\title{
Article \\ Influence of Optic Cable Construction Parts on Recovery Process after Gamma Irradiation
}

\author{
Zuzana Šaršounová ${ }^{1,2}$, Vít Plaček ${ }^{1, *}$, Václav Prajzler ${ }^{3}{ }^{10}$, Kateřina Masopustová ${ }^{3}$ and Petr Havránek ${ }^{1}$ \\ 1 ÚJV Řež, a. s., Hlavní 130, Řež, 25068 Husinec, Czech Republic; zuzana.sarsounova@ujv.cz (Z.Š.); \\ petr.havranek@ujv.cz (P.H.) \\ 2 Department of Electrotechnology, Faculty of Electrical Engineering, Czech Technical University in Prague, \\ Technicka 2, 16627 Prague, Czech Republic \\ 3 Department of Microelectronics, Faculty of Electrical Engineering, Czech Technical University in Prague, \\ Technicka 2, 16627 Prague, Czech Republic; vaclav.prajzler@fel.cvut.cz (V.P.); masopkat@fel.cvut.cz (K.M.) \\ * Correspondence: vit.placek@ujv.cz
}

check for updates

Citation: Šaršounová, Z.; Plaček, V.; Prajzler, V.; Masopustová, K.; Havránek, P. Influence of Optic Cable Construction Parts on Recovery Process after Gamma Irradiation. Energies 2022, 15, 599. https:// doi.org/10.3390/en15020599

Academic Editors: Simone Vincenzo Suraci, Xavier Colin and Davide Fabiani

Received: 17 November 2021

Accepted: 11 January 2022

Published: 14 January 2022

Publisher's Note: MDPI stays neutral with regard to jurisdictional claims in published maps and institutional affiliations.

Copyright: (C) 2022 by the authors. Licensee MDPI, Basel, Switzerland. This article is an open access article distributed under the terms and conditions of the Creative Commons Attribution (CC BY) license (https:// creativecommons.org/licenses/by/ $4.0 /)$.

\begin{abstract}
Fibre optic cables are widely used as communication cables in Instrumentation and Control (I\&C) systems. In the case of nuclear power plants (NPPs), using optic cables in mild environments outside of containment areas are very common. However, at present, there is a need for fibre optic cables to be used in containment areas, i.e., with radiation. An optical fibre consists of a highly transparent core that possesses a higher refractive index than the surrounding transparent cladding, which possesses a lower refractive index. Most optical fibres are manufactured from glass (silica with required dopants) which is created at high temperatures from the reaction between gasses. The glass used in optical fibres is sensitive; it becomes dark during exposure to radiation, which compromises the optic functions. That is why there has been a slow infiltration of optic cable in NPP containment areas. Radiation resistant optic fibres have been developed. Although these fibres are called "radiation resistant," they go through a darkening process (absorbance increase) as well, but not as quickly. Immediately after the irradiation has stopped, a recovery process starts in the glass structure. During this period, optical losses of the glass improve, but not to the original level as before the irradiation. During the testing of optic cables for the installation in nuclear power plant containment areas, we observed an unusual recovery process. In the beginning, a healing effect was observed. However, after a few days of recovery, the healing process stopped, and the trend changed again as a worsening of the optical properties was observed. This paper describes experiments which explain the reasons for such an unexpected behaviour.
\end{abstract}

Keywords: fibre optic; irradiation; attenuation; recovery process; nuclear power plants

\section{Introduction}

Fibre optic cables are commonly used at Nuclear Power Plants (NPPs) in I\&C systems but they are usually used outside of containment areas and in mild environments. For example, the Czech NPP Dukovany, a Pressurized Water Reactor (PWR) type with four $440 \mathrm{MW}$ units, has almost 1500 optical cables installed with a total length of $150 \mathrm{~km}$. All the optic cables are situated in areas outside radiation zones. Nevertheless, currently there is a requirement to use optic cables in NPP containment areas, which means in locations with radiation. The values of radiation dose rate depend on the location within the NPP containment. It may vary from almost nothing up to $1 \mathrm{~Gy} / \mathrm{h}$ near the steam generator or main circulating line [1] which gives a total radiation dose of up to $350 \mathrm{kGy}$ during a 40-year lifetime. Hence, it is very important to know where the cables are expected to be installed, at which level of radiation and what the expected service lifetime is forecasted to be.

The application of fibre optic cables on systems important to the safety in NPPs requires their qualification. For this reason, the international standard for qualification 
IEEE 1682 [2] has existed since 2011. The qualification process (demonstration that the equipment is able to function within its required accuracy and performance requirements in all required operational and environmental conditions [2,3]) is described in our previous paper [4] and is briefly summarized in Section 2.

Radiation is the most significant environmental stressor in a NPP environment for optical fibres [2,4], which causes fibre darkening, i.e., a dramatic optical attenuation increase and a loss of functionality. Radiation leads to the formation of colour centres in the glass structures [5-11], which cause a change in the absorption characteristics leading to the colouration of the fibre. This effect is so significant that it can lead to the "blinding" of an optical fibre with attenuations in tens of $\mathrm{dB}$ per $\mathrm{km}$ depending on the radiation dose. This Radiation-Induced Attenuation (RIA) phenomenon is caused by the generation through ionization or displacement damages of point defects in both silica-based fibre core and fluorine-doped cladding [8]. After the irradiation has stopped, glass fibre goes through a "recovery process" (otherwise relaxation) [2,12], when the glass becomes transparent again, i.e., attenuation decreases $[10,13]$. However, this process is not completely reversible. The extent of recovery can be affected, among others, by time and temperature after the radiation ageing. It was previously shown that the optical losses of the fibres are heavily dependent upon dopants and defects in the optical fibres. Therefore, different fibre compositions will be affected differently [14].

UJV Rez a.s. testing laboratory has qualified two types of optic cables with different fibres for the installation in NPPs. Results concerning the ionizing radiation are as follows:

- Simplex and breakout cables with multi-mode (MM) fibres increased their attenuation during irradiation. Nevertheless, during the next 30 days of the recovery process, the attenuation continuously decreased as expected [4]. The trend is displayed in Figure 2 on the blue line "Expected attenuation recovery". The fibre used was a MM 62.5/125, J-fiber, radiation-hardened fibre, MIL-PRF-49291/6C. The Simplex cable consisted of fibre, buffer, aramid yarn and cord. The breakout cable consisted of 24 simplex cables, a central strength member, PE tapes, additional aramid yarn and an outer jacket. The initial measured values of attenuation were $1 \mathrm{~dB} / \mathrm{km}$ at $1300 \mathrm{~nm}$, and the highest measured value after irradiation with a dose of $55 \mathrm{kGy}$ was $40 \mathrm{~dB} / \mathrm{km}$.

- The situation for gel-filled loose tube single-mode (SM) cables was quite different. Attenuation decreased (recovered) a few days after the irradiation. After this short period, the trend changed and attenuation increased to a value well above the attenuation just after the irradiation; see red line in Figure 2. The fibre used was a SM 9/125 $\mu \mathrm{m}$, ITU-T G.652, J-fiber, radiation hardened fibre. The construction of the cable is shown in Figure 1. The initial measured values of attenuation were $0.4 \mathrm{~dB} / \mathrm{km}$ at $1310 \mathrm{~nm}$, and the highest measured value just after irradiation with a dose of $55 \mathrm{kGy}$ was $51 \mathrm{~dB} / \mathrm{km}$. During the following process, when we expected to observe signs of recovery, the attenuation increased up to $146 \mathrm{~dB} / \mathrm{km}$. Similar values were also measured at $1550 \mathrm{~nm}$.

This atypical recovery behaviour was observed only on loose tube cables with SM fibres placed in tubes. Two kinds of this cable construction were tested-the small one contained only four fibres in one tube while the other cable contained 12 tubes with 12 fibres per tube. The gel was used as a waterproof barrier in the cable tubes, as depicted in Figure 1.

Such high attenuation values disqualify these SM fibres for use in NPP containment environments. Therefore, additional experiments were performed trying to explain the reasons for such an unexpected behaviour. 


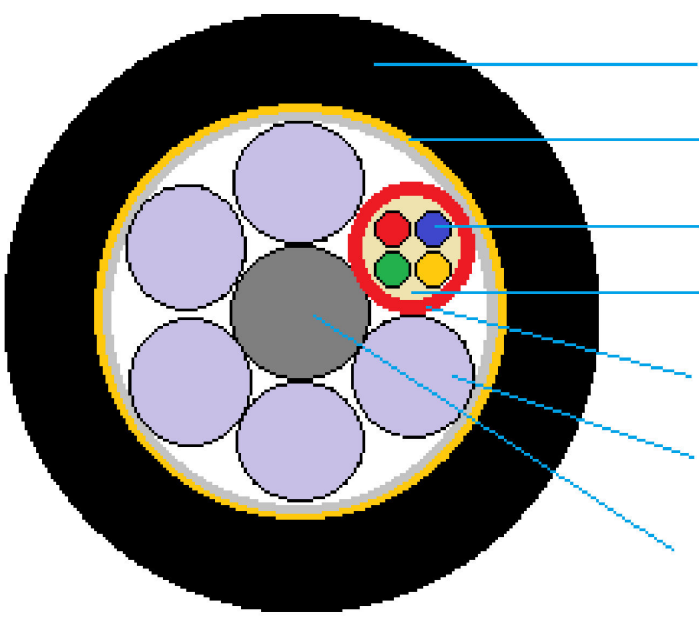

\section{Outer jacket}

Aramid yarn

Optical fiber

Jelly

Loose tube

Filler

Central strength member

Figure 1. Schematic cross-section of the optic cable tested with four fibres. The number of fibres in the tests were four (as in the drawing) and/or 12 in each tube.

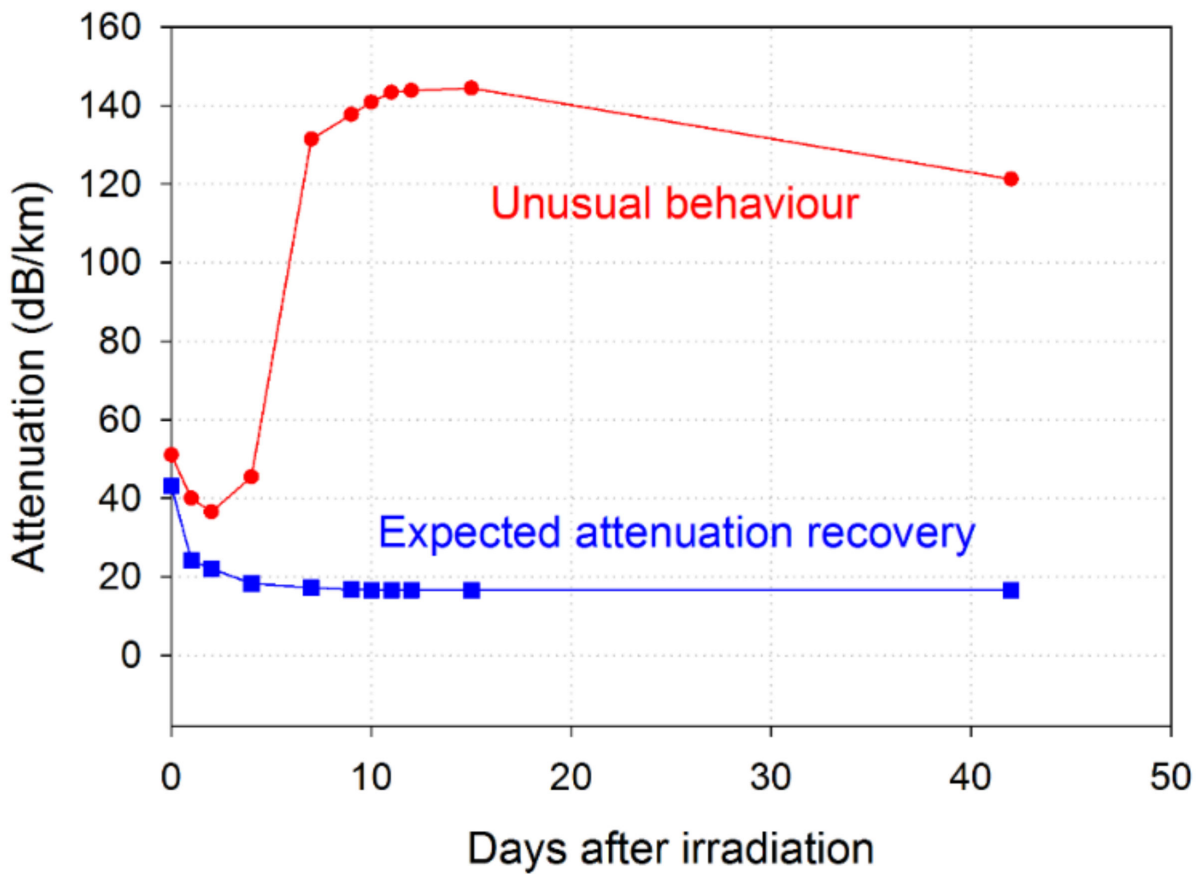

Figure 2. Attenuation measurement performed at $1300 \mathrm{~nm}$ (blue line, MM) and/or $1310 \mathrm{~nm}$ (red line, SM) after optic cable irradiation. During the recovery process after the irradiation has stopped, attenuation decreased, i.e., improvement of optical properties is expected. Some types of fibre optic cables exhibit an inverse recovery process after a few days of the recovery period. This Figure has already been published in [4].

\section{Methodology}

To understand tests which are performed, the qualification process for nuclear power plants applications according to the standard [2] is briefly described. The text is partially taken from our previous paper [4] which has been repeated here for a better understanding of the presented results. Basically, all of the stressors causing age-related degradation need to be simulated. The required procedure to qualify fibre optic cables is as follows:

- Accelerated thermal ageing simulates the long-term operation at elevated temperatures.

- Accelerated radiation ageing simulates the stress encountered from the influence of ionizing radiation during operation. 
- $\quad$ Simulation of mechanical stress.

- Simulation of accident conditions.

- Throughout the qualification, functional properties shall be monitored. The most important property is attenuation.

Only the irradiation was described in this paper because it is the most important factor which influences fibre optic cable attenuation $[2,4]$.

\subsection{Irradiation}

The radiation ageing was simulated by irradiation at room temperature and at atmospheric pressure with a ${ }^{60} \mathrm{Co}$ gamma-ray source with a dose rate higher than what would normally be found in a real environment. The dose was determined by alanine/ESR dosimetry and stated as dose in Si [15]. During irradiation, the samples were coiled on a perforated stainless-steel cylinder with a diameter of $0.4-0.8 \mathrm{~m}$, while the cobalt source was in the middle of the cylinder. The energy of the gamma photons/rays from the ${ }^{60} \mathrm{Co}$ were 1.17 and $1.33 \mathrm{MeV}$, respectively. The fibre optic cables which were tested did not have any metal parts. Thus, the total dose was homogeneously distributed within the whole sample. Cable ends with connections were placed in a position with a much lower dose rate or they were shielded. For an easier survey, only mean values of total dose and dose rates are provided, without uncertainties. After irradiation, the cables were straightened and coiled at least once at the in-service minimum bend radius of the cable, which was for the cables being tested, $20 \times$ cable diameter (20D). Such mechanical stress is required by the standard IEEE 1682 [2]. However, bending was not applied in additional experiments.

Total doses were between 10 and $50 \mathrm{kGy}$ and represent expected operational doses of the possible fibre optic cable installation in a respective NPP. Applied doses and dose rates are mentioned in the text below.

\subsection{Attenuation Measurement}

To measure attenuation with a reasonable rate of uncertainty, the optical route should be at least $100 \mathrm{~m}$ long. To increase the optical length of the cable, individual fibres within the cable can be connected to a series by fusion splicing. Nevertheless, it must be ensured that these fusions and connections do not influence the results.

MM cables were measured at wavelengths $850 \mathrm{~nm}$ and $1300 \mathrm{~nm}$, and SM cables at $1310 \mathrm{~nm}$ and $1550 \mathrm{~nm}$ (in a few cases at $1625 \mathrm{~nm}$ ). Differences in results were discussed in the previous paper [4] and are insignificant. Therefore, in this paper, only the results at the wavelength $1550 \mathrm{~nm}$ are shown.

During the experiments, we used two attenuation-measuring methods:

- $\quad$ LSPM-Light Source and Power Meter (EN/IEC 60793-1-40-method B Insertion Loss)

- OTDR - Optical Time Domain Reflectometer (EN/IEC 60793-1-40—method C Backscattering)

Attenuation was measured before irradiation, in some cases during the irradiation, just after the irradiation and then during the following 30 days when the samples were stored at room temperature. The measuring devices were connected to the tested samples during the 30 days of the recovery process and could not be easily used for another experiment. Therefore, only a limited number of experiments was performed and some of our conclusions were not proven on more samples. The main goal was to find the causes of the inverse recovery process.

Measuring the attenuation was performed discretely, i.e., in specific intervals or continuously to monitor its trend. In the case of discrete measurement, the equipment was connected/disconnected, which caused imperfect results.

\section{Experimental Plan}

In the previous work [4], the experiments mainly verified the individual steps of the qualification to exclude the probability of mistakes during the qualification process. The summarization list of the experiments performed and their results are as follows: 
- Influence of connector and fusions irradiation

To check the possible influence of connectors and fusion splicing, the same samples were irradiated in such a way that the ends of the cable were shielded and/or pulled out of the irradiation facility. There was no difference between the ends with connectors that were pulled out (i.e., not irradiated) or were slightly irradiated which was the case from previous testing.

- SM fibres with different dopants

In this experiment, we tested only the fibre samples to check the role of Ge dopants on the inverse recovery process. We found that the dopants used did not have any effect on radiation-resistant fibres (two types were tested) and there is no increase in attenuation during the recovery process if only the fibres alone are irradiated.

\section{- Thermal ageing}

The first step of qualification is a simulation of long-term thermal ageing. In accordance with the standard [2], thermal ageing is simulated using the Arrhenius approach, which assumes that a short-term simulation of thermal ageing at higher temperatures causes the same degradation as long-term ageing at lower temperatures. To check the possible influence of thermal ageing, the cable was irradiated also without any thermal pre-treatment. Both cables, thermally aged as well as without thermal treatment, exhibited the same inverse recovery process.

- Mechanical stress after irradiation

Mechanical stress is caused by straightening and bending immediately after the irradiation. Such a procedure is required by the standard and simulates possible mechanical stresses encountered in the operation of an NPP and during some accidents that may occur. Therefore, cables coiled around a mandrel after irradiation as well as samples without mechanical stress were measured during the recovery process. There was no difference; i.e., the mechanical stress is not the reason for the inverse recovery behaviour.

- Dose rate effect

The cable and fibre were irradiated alone at a dose rate of $1.45 \mathrm{kGy} / \mathrm{h}$ and compared with the cable and fibre sample irradiated at $0.0173 \mathrm{kGy} / \mathrm{h}$, i.e., approx. 100 times lower dose rate. The total dose was around $15 \mathrm{kGy}$. The attenuation increasing during and after irradiation was approximately two times higher when the whole cable was irradiated compared to when the fibre was irradiated on its own. The inverse recovery effect (in the case of cables) was much higher when irradiated at a higher dose rate.

The experiments presented did not explain the nature of the inverse recovery process. Nevertheless, we could exclude that the inverse recovery process is associated with some necessary procedure during the qualification process. Based on our results, we can also state that this phenomenon is not caused by the fibreglass material itself because when the fibres were irradiated on their own, the inverse recovery process did not appear. The next set of experiments described in this paper was focused on studying the possible effect of total radiation dose and the differences in cable construction.

\section{Results and Discussion}

The cable which was found to have the inverse recovery process is a gel-filled loose tube SM cable. Hence, we checked the potential influence and interaction of the material surrounding the fibre, i.e., the coating on the fibre, gel and the loose tube.

\subsection{Influence of the Coating}

To test any possible interactions between the coating and the SM fibre itself, a few fibre samples with different coating colours (red and blue) were tested.

Both red and blue samples were irradiated with a total dose of $15 \mathrm{kGy}$. The attenuation decrease after the irradiation was the same for both samples. The coating did not affect the inverse recovery process. 


\subsection{Influence of the Loose Tube and Number of Fibres in the Tube}

Cables with the same construction as shown in Figure 1 with gel-filled Polybutylene Terephthalate (PBT) tubes manufactured by two different suppliers were tested. One tube had a partially porous wall, while the second one was without any defects (Figure $3 a, b$ ).

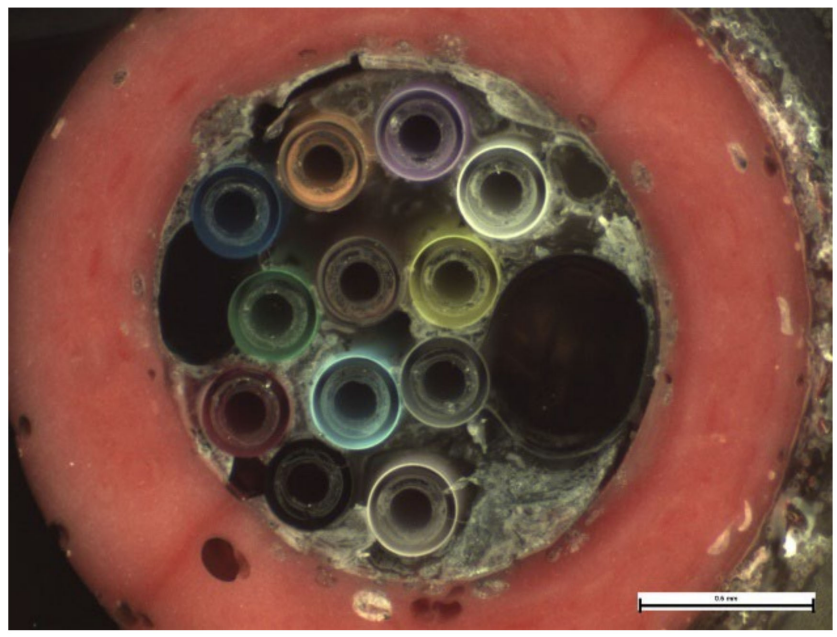

(a)

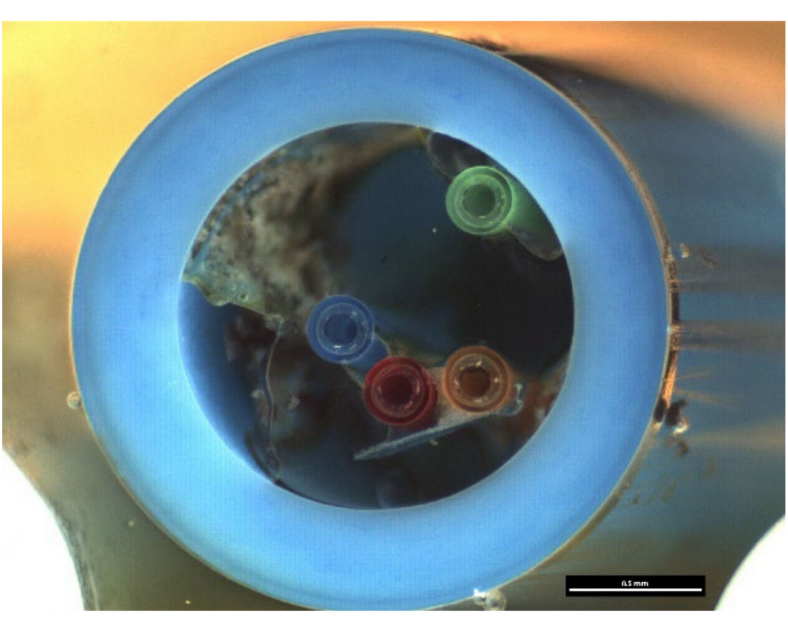

(b)

Figure 3. (a) Gel-filled loose tube cable: red porous tubes. Tube diameter is $2.3 \mathrm{~mm}$ (inserted scale of the picture is $0.5 \mathrm{~mm}$ ). The same tube was tested with both four fibres as well as 12 fibres running through the cables. (b) The non-porous gel-filled tube with four fibres. Tube diameter is $2.2 \mathrm{~mm}$ (the scale of the picture is $0.5 \mathrm{~mm}$ ).

In addition, two cable dimensions of porous tube cable were parallelly tested. One cable was with four fibres inside the loose tube and one cable with 12 fibres per tube with almost the same diameter. After the irradiation with a dose of $17.4 \mathrm{kGy}$ and a dose rate $0.60 \mathrm{kGy} / \mathrm{h}$, the sample with only four fibres exhibited a stronger inverse recovery behaviour (see Figure 4).

Summary of the tube influence:

- Two PBT loose tubes manufactured by different suppliers were tested. One "imperfect" tube had pores in the wall, while the second one was more homogeneous. Both samples were accounted for in the inverse recovery process. This suggests that the mechanism responsible for the investigated inverse recovery process is due to the gel.

- When the same type of gel-filled loose tubes with 12 fibres and with only four fibres were irradiated, the attenuation line during inverse recovery was not identical. The cable sample with only four fibres inside the tube showed a stronger inverse effect. The effect shows again that the gel may be the main reason for the inverse recovery process. The amount of gel in the tube with four fibres was much more than in the tube with 12 fibres.

\subsection{Influence of the $\mathrm{Gel}$}

The above-mentioned experiments indicate that the component causing the inverse recovery is the gel. To prove this, we tested cables with porous tubes with gel inside (4 and 12 fibres) and without gel (dry type non-porous tube cable, with four fibres in a tube). After irradiation with a dose of $17.4 \mathrm{kGy}$ and a dose rate of $0.60 \mathrm{kGy} / \mathrm{h}$, the attenuation started to decrease. In the case of the dry type, once the irradiation stopped, the attenuation continuously decreased while the samples with gel exhibited an inverse recovery process (Figure 4). To exclude the possibility of a local defect, OTDR measurements were performed. They did not indicate any local disturbances within the measured path. The attenuation had the same homogeneous level within the length of the entire fibre. 


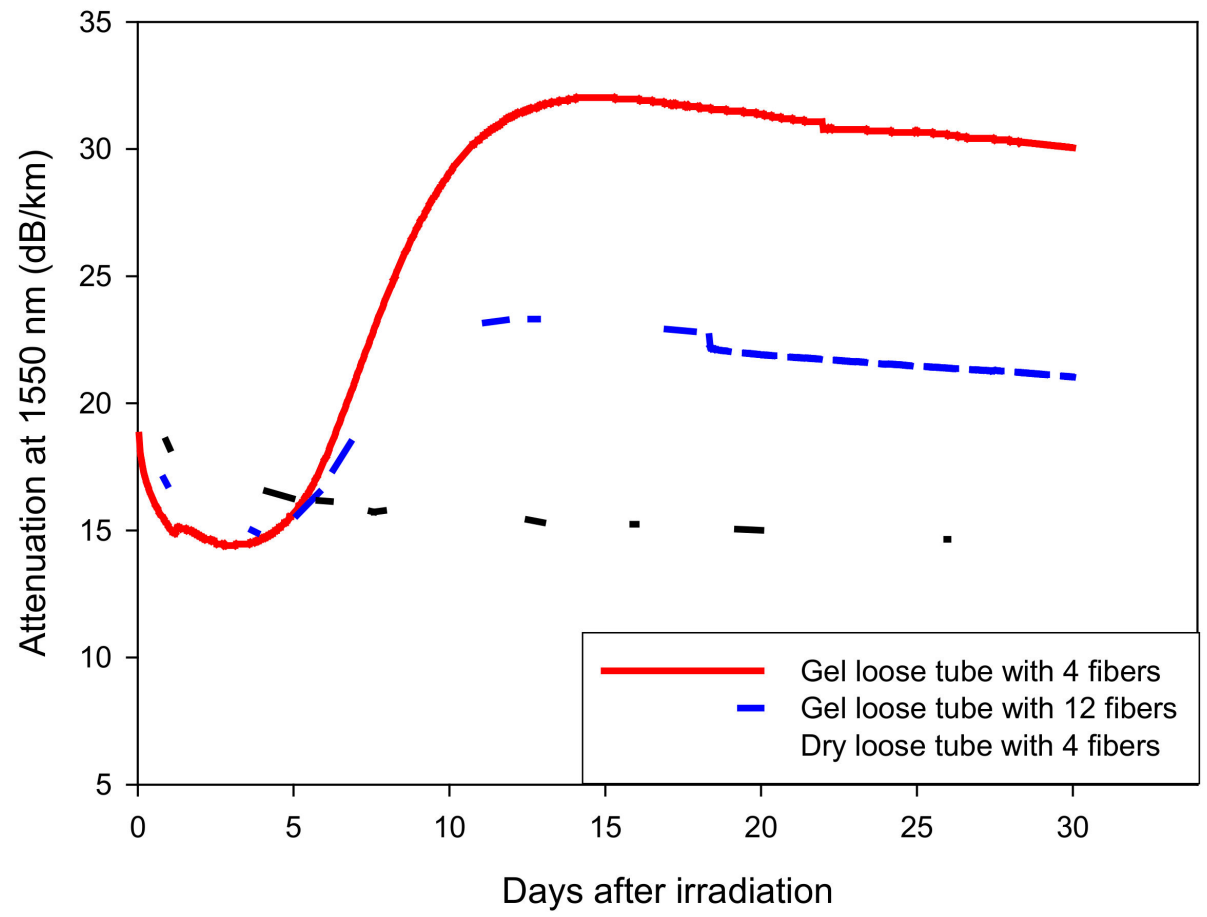

Figure 4. Influence of the gel. Tests were performed on cable samples with the gel (4 or 12 fibres in one tube) and without the gel-dry type with four fibres in a tube. Measurements were not carried out continuously. The test equipment was connected/disconnected for each measurement, which caused small drops and imperfections visible on the curves.

Based on all these results, we can clearly state that the component responsible for the inverse recovery process is the gel.

The Shanghai Honghui Optic Communication Tech produces gel under the name LT-600. The exact chemical composition is unknown. Information from the material safety data sheet is the following: mineral oil $93 \%$, thermoplastic rubber $6.3 \%$, anti-oxidant, polyethylene $0.7 \%$.

There are a few hypotheses on how a gel can influence or cause an inverse attenuation effect after irradiation. Some of them are as follows:

- Gel expands its volume due to gaseous radiolytic product formation, inner crosslinking and chemical reactions in the gel, resulting in mechanical stress.

- Gel may shrink which results in mechanical stress.

- $\quad$ Radiolytic products react with the fibre's coating or diffuse into the glass.

To test the hypotheses about the stress due to volume increase/decrease, we irradiated two samples: a non-treated loose tube and a perforated sample enabling gel to leak. In approximately $20 \mathrm{~cm}$ distances, the loose tube was perforated by a fibre optic buffer stripper. In the places of perforation, a small outflow of gel was observed. Continuity of the optical route was controlled before irradiation by OTDR, to ensure that there was no route interruption caused by stripping. However, this simple experiment did not show any improvement in the inverse recovery process.

A possible explanation is that radiation creates hydrogen species that are able to diffuse from the gel through the fibre coating up to the fibre core, interacting with created point defects (such as non-bridging oxygen hole centres $\mathrm{Si}-\mathrm{O}$ ). They can create $\mathrm{Si}-\mathrm{OH}$, that are associated with absorption peaks, especially at $1380 \mathrm{~nm}$ [8]. The delay between the irradiation and the observed effects may be explained by the diffusion time of hydrogen species in the fibre. The hydrogen can be, on one hand, easily loaded at room temperature in silica-based fibres, but on the other hand, it will also quickly diffuse out. This fact 
renders the treatment efficiency dependent on the time delay between the treatment and the irradiation $[8,14]$.

To check this possibility, SM fibre in the tube with gel was irradiated with a gammaray irradiation dose of $1.12 \mathrm{kGy} / \mathrm{h}$ for $9 \mathrm{~h}$. The total gamma radiation dose was $10.1 \mathrm{kGy}$. During irradiation, we measured optical losses at three wavelengths, 1310, 1550 and $1625 \mathrm{~nm}$, using Insertion \& Reflection Loss Meter JCIRL-300 with detector Jericore, and the obtained results are shown in Figure 5a. The measurements show that the optical losses increase with increasing gamma-ray dose and optical losses at $1310 \mathrm{~nm}$ are higher than at wavelengths 1550 and $1625 \mathrm{~nm}$. We can observe lower values of the optical losses after $390 \mathrm{~min}$ and $450 \mathrm{~min}$ (at the wavelength $1310 \mathrm{~nm}$ ) during the gamma rays' irradiation. These lower values are caused by how the measurements were taken, which required the connection/disconnection of the applied optical sources and the optical fibres which were measured.

a)

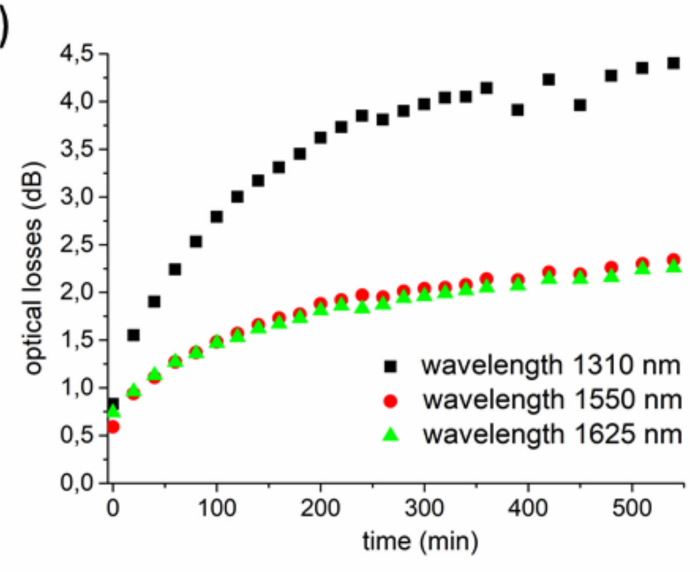

b)

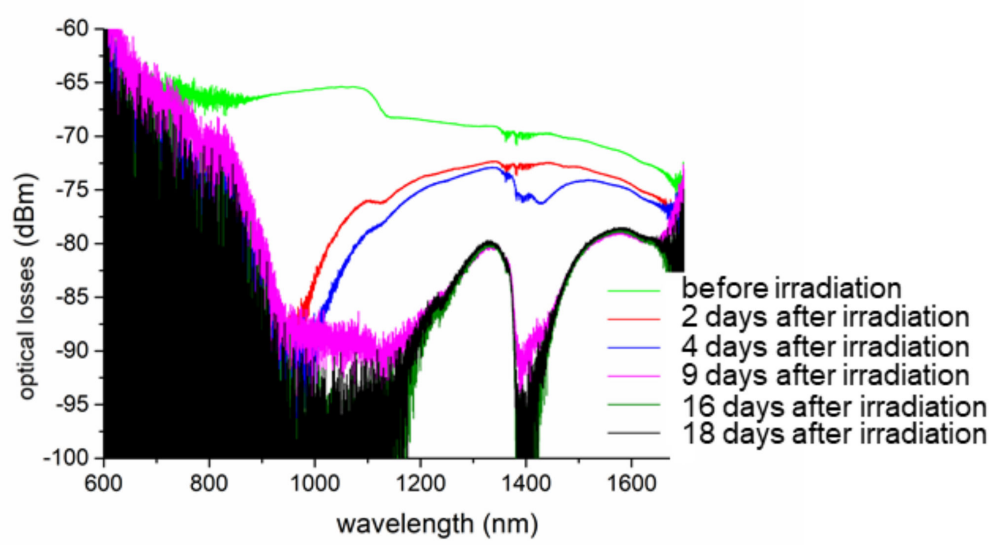

Figure 5. (a) Optical losses of the optical fibre during gamma ray irradiation $1.12 \mathrm{kGy} / \mathrm{h}$ for $9 \mathrm{~h}$. (b) Spectral characteristic of the optical fibre measured after gamma ray irradiation (10.1 kGy).

After gamma-ray irradiation was stopped, we measured the optical spectrum. The measurement was done using an optical spectrum analyzer YOKOGAWA AQ6370C in the range from 600 to $1700 \mathrm{~nm}$ and we used the optical source HL-2000 FHSA (Ocean Optics) with a typical output power of $8.4 \mathrm{~mW}$. First, we measured optical losses pristine optical fibre before gamma-ray irradiation, then we repeated measurement at 2, 4, 9, 16 and 18 days after irradiation. The results which were obtained are shown in Figure 5b.

Figure $5 \mathrm{~b}$ shows an increase in optical losses after irradiation. We observed that 9 days after irradiation, the increase of the optical losses stopped. Figure $5 \mathrm{~b}$ also shows that optical losses increased higher at wavelengths around $1380 \mathrm{~nm}$ than at other wavelengths. This is due to an increase in the content of hydroxyl groups ( $\mathrm{Si}-\mathrm{OH})$ at an attenuation peak of $1385 \mathrm{~nm}$ and the next reason for this increment of the optical losses could be to acrylate coating due to the radiation induced radiolytic hydrogen from the polymer coating diffusing to the fibre core [14].

\section{Conclusions}

When fibre optic cables are exposed to gamma-irradiation, fibre darkening can be caused with an attenuation increase. After the irradiation has stopped, the fibre goes through a recovery process with an attenuation decrease. This effect is widely known and was also proved in the UJV Řež testing laboratory when multi-mode (MM) fibre optic cables were irradiated. However, after single-mode (SM) cable irradiation, the attenuation decreased as expected for only a few days. After this short period of time, attenuation changed dramatically, increasing to a value well above the attenuation just after the irradiation had finished. We call this phenomenon "inverse recovery process", and it may play a negative role in the use of fibre optic cables in nuclear power plants. 
A lot of experiments were performed to find the reason for this effect [4]. This paper describes an additional set of experiments.

It has been proved that the component responsible for the inverse recovery process is the gel. The main evidence is based on the following results:

- Dry loose tube without gel after irradiation goes through continuously healing effects with attenuation recovery, while the same loose tube which contains gel exhibits an inverse recovery process.

- Loose tube cable with a larger amount of gel has a much stronger inverse recovery process than a sample of loose tube cable with a smaller amount of gel.

- Changing other parameters, like mechanical stress after irradiation, thermal ageing before irradiation, different fibres, different coatings, total dose and the applied radiation dose rate had no effect on the recovery process.

Some hypotheses on how the gel may influence the recovery are suggested below:

- Gel expands its volume due to gaseous radiolytic products forming, inner cross-linking and chemical reactions in the gel, resulting in mechanical stress.

- Gel may shrink which results in mechanical stress.

- Radiolytic products react with the fibre coating or diffuse into the glass.

A comparative experiment with fibres suspended in gel in a high-quality tube (Figure $3 b$ ) and in a perforated tube enabling the gel to leak was performed. The presence or absence of mechanical stress did not show any of the inverse recovery processes.

A possible explanation is that radiation creates hydrogen species, slowly diffusing from the gel through the fibre coating up to the fibre core and creating $\mathrm{Si}-\mathrm{OH}$, which are associated with absorption at $1385 \mathrm{~nm}$. The presented work is more or less experimental in nature with the main goal to explain the reasons for the inverse recovery process and for fibre optic cables to be safely installed within nuclear power plant containment systems. Additional experiments studying the gel performance under irradiation, hydrogen detection and other radiolytic products detection are planned. These additional experiments will be supported by theoretical investigations concerning hydrogen species diffusion.

Author Contributions: Z.Š., V.P. (Vít Plaček) and V.P. (Václav Prajzler) conceived and designed the experiments; K.M. and P.H. performed the experiments; Z.Š., V.P. (Vít Plaček) and V.P. (Václav Prajzler) analyzed the data and wrote the paper. All authors have read and agreed to the published version of the manuscript.

Funding: Our research has been supported by the Czech Technical University in Prague with the SGS program (SGS20/175/OHK3/3T/13) and Centre of Advanced Applied Natural Sciences", Reg. No. CZ.02.1.01/0.0/0.0/16_019/0000778, supported by the Operational Program Research, Development and Education, co-financed by the European Structural and Investment Funds and the state budget of the Czech Republic.

Institutional Review Board Statement: Not applicable.

Informed Consent Statement: Not applicable.

Data Availability Statement: The data that support the findings of this study are available from the corresponding author upon reasonable request.

Acknowledgments: Test samples were kindly provided by GOC Co., Ltd. (Fibre optic cable manufacturing company, Gwangju, Korea). Special thanks to colleagues from VŠB (Technical University of Ostrava, Faculty of Electrical Engineering and Computer Science, Department of Telecommunications) for the initial cooperation and measurement.

Conflicts of Interest: The authors declare no conflict of interest. 


\section{References}

1. Anonymous. Technical Basis for Commendable Practices on Ageing Management—SCC and Cable Ageing Project (SCAP), Final Report; NEA/CSNI/R(2010)5; OECD Nuclear Energy Agency: Paris, France, 2010.

2. IEEE 1682-2011; Standard for Qualifying Fiber Optic Cables, Connections, and Optical Fiber Splices for use in Safety Systems in Nuclear Power Generating Stations. IEEE: Piscataway, NJ, USA, 2013.

3. No. SSG-69; Equipment Qualification for Nuclear Installations; IAEA Safety Standards, Specific Safety Guide. IAEA: Vienna, Austria, 2021.

4. Konečná, Z.; Plaček, V.; Havránek, P. Unusual Attenuation Recovery Process after Fiber Optic Cable Irradiation. IOP Conf. Ser. Mater. Sci. Eng. 2017, 266, 012005. [CrossRef]

5. Morana, A.; Girard, S.; Cannas, M.; Marin, E.; Marcandella, C.; Paillet, P.; Périsse, J.; Macé, J.-R.; Boscaino, R.; Nacir, B.; et al Influence of neutron and gamma-ray irradiations on rad-hard optical fiber. Opt. Mater. Express 2015, 5, 898-911. [CrossRef]

6. Sandhu, A.K.; Singh, S.; Pandey, O.P. Gamma ray induced modifications of quaternary silicate glasses. J. Phys. D Appl. Phys. 2008, 41, 165402. [CrossRef]

7. Girard, S.; Richard, N.; Ouerdane, Y.; Origlio, G.; Boukenter, A.; Martin-Samos, L.; Paillet, P.; Meunier, J.-P.; Baggio, J.; Cannas, M.; et al. Radiation Effects on Silica-Based Preforms and Optical Fibers-II: Coupling Ab initio Simulations and Experiments. IEEE Trans. Nucl. Sci. 2008, 55, 3508-3514.

8. Campanella, C.; De Michele, V.; Morana, A.; Mélin, G.; Robin, T.; Marin, E.; Ouerdane, Y.; Boukenter, A.; Girard, S. Radiation Effects on Pure-Silica Multimode Optical Fibers in the Visible and Near-Infrared Domains: Influence of OH Groups. Appl. Sci. 2021, 11, 2991. [CrossRef]

9. London, Y.; Sharma, K.; Diamandi, H.H.; Hen, M.; Bashan, G.; Zehavi, E.; Zilberman, S.; Berkovic, G.; Zentner, A.; Mayoni, M.; et al. Opto-Mechanical Fiber Sensing of Gamma Radiation. J. Lightwave Technol. 2021, 39, 6637-6645. [CrossRef]

10. Esposito, F.; Srivastava, A.; Campopiano, S.; Iadicicco, A. Radiation Effects on Long Period Fiber Gratings: A Review. Sensors 2020, 20, 2729. [CrossRef] [PubMed]

11. Kashaykin, P.F.; Tomashuk, A.L.; Vasiliev, S.A.; Ignatyev, A.D.; Shaimerdenov, A.A.; Ponkratov, Y.; Kulsartov, T.V.; Kenzhin, Y.A.; Gizatulin, S.K.; Zholdybayev, T.K.; et al. Radiation resistance of single-mode optical fibres with view to in-reactor applications. Nucl. Mater. Energy 2021, 27, 100981. [CrossRef]

12. Senior, J.M.; Jamro, M.Y. Optical Fiber Communications: Principles and Practice, 3rd ed.; Person Education: London, UK, 2009.

13. Vašínek., V.; Hájek, L.; Bednárek, L.; Vanderka, A.; Hlavinka, T.; Marcinka, O. Influence of gamma radiation on optical fibers and devices. In Proceedings of the International Conference on Photonics Solutions 2015, Hua Hin, Thailand, 6-8 July 2015.

14. Stone, J. Interactions of Hydrogen and Deuterium with Silica Optical Fibers: A Review. J. Lightwave Technol. 1987, 5, 712-733. [CrossRef]

15. ISO/ASTM 51261:2013; Practice for Calibration of Routine Dosimetry Systems for Radiation Processing; Confirmation 2018. ISO: Geneva, Switzerland, 2013. 